\title{
Recent respiratory infection and the risk of myocardial infarction
}

\author{
T C Clayton, N E Capps, N G Stephens, J A Wedzicha, T W Meade
}

Heart 2005;91:1601-1602. doi: 10.1136/hrt.2004.046920

$M$ ounting interest over the past 10 or 15 years in the possible role of infection in the risk of myocardial infarction (MI) has mainly centred on serological evidence of infection that often has occurred several years or months previously. ${ }^{1}$ However, recent rather than distant infection may be more strongly associated with coronary events, as shown by the excess of coronary deaths in the winter months not accounted for by the concomitant increase in deaths from respiratory disease. ${ }^{2}$ The most suggestive evidence comes from two studies carried out through the General Practice Research Database (GPRD). ${ }^{3}$ In the first, the adjusted odds ratio (OR) for MI within 10 days of a respiratory infection was 3.0 (95\% confidence interval (CI) 2.1 to $4.4, \mathrm{p}<0.0001$ ) and was nearly 4.0 within five days of infection. In the second study, incidence ratios for MI were $4.95,3.20,2.81$, and 1.95 at days $1-3,4-7,8-14$, and $15-28$ after infection, respectively. We therefore carried out a clinical case-control study, collecting information directly from patients admitted with MI and matched controls through a structured questionnaire to try to confirm or refute the indications of the GPRD studies based on documentary information.

\section{METHODS}

Cases were recruited from patients admitted with a clinical diagnosis of MI to the coronary care units at two hospitals. Potential controls were recruited from neighbouring general practices matched for age, sex, and area deprivation score. In total 119 cases and 214 matched controls were recruited. The primary definition of respiratory infection was taken from the questionnaire data as: (1) any two of runny nose, stuffy or blocked nose, sore throat, hoarseness or general cold symptoms; or (2) any two of cough, sputum, or sputum colour change starting within the previous month. Participants were also asked about any chest pains on breathing and any fever (fever, high temperature, or night sweats). Conditional logistic regression was used to assess the association between symptoms of infection and MI. Results were adjusted for smoking status and history of angina.

\section{RESULTS}

The mean age of participants was 63 years and $84 \%$ were men. All cases were interviewed within a week of admission, with $88 \%$ seen within the intended time period of four days. There was no evidence of an association between the primary definition of respiratory infection and MI (adjusted OR 1.0, 95\% CI 0.5 to $1.9 ; \mathrm{p}=0.98$ ) (table 1 ). However, there was an unexpectedly high OR for the association of chest pain on breathing with infarction (adjusted OR 17.5, 95\% CI 3.6 to 85.6; $\mathrm{p}<0.0001)$. For fever, there was also a strong association (adjusted OR 5.9, 95\% CI 2.0 to 16.8; $\mathrm{p}=0.0004)$. Further analyses restricted to those participants reporting symptoms of both chest pain and fever, and thus with a seemingly certain diagnosis of lower respiratory infection, showed that all seven participants reporting both together were cases $(p=0.001)$. For five of these cases, the symptoms first appeared at least two weeks before the index event. To reduce the likelihood that chest pain might have been due to the early onset of MI, the data were also analysed by omitting patients who had chest pain on breathing (without fever) within the preceding week. There remained a strong association between pain and infarction (adjusted OR 9.3, 95\% CI 1.5 to 56.4; $\mathrm{p}=0.009)$. There were also strong associations between chest pain on breathing or fever and symptoms of the primary definition of respiratory infection. Over time, the OR for chest pain or fever was 10.2 (95\% CI 3.4 to 30.7 ) at $1-2$ weeks before the MI, 8.2 (95\% CI 2.3 to 29.5 ) at $3-4$ weeks, and 0.9 (95\% CI 0.4 to 2.1 ) at $\geqslant 1$ month.

\section{DISCUSSION}

The numbers of participants in the study were not large but were nevertheless sufficient to show unexpectedly strong associations of reported chest pain on breathing with infarction. Recall bias by cases, who may have been more likely than controls to have recorded symptoms, has to be considered but this would have to have been extreme to account for the full extent of the odds ratios presented. Another possibility is that chest pain might in some cases have been a premonitory symptom of the infarct. Against this explanation is, firstly, that the association was still strong after cases whose pain had started within the week immediately preceding the MI were omitted. Secondly, pleuritic pain on breathing is not well documented as a premonitory symptom of MI and there was no evidence that the excess of chest pain in cases was caused by angina. Lastly, the association of chest pain with symptoms of the primary definition suggests that chest pain was part of the range of symptoms occurring in the respiratory infections reported. Data from the general practice based IMS Disease AnalyserMediPlus database (IMS Health, London, UK) showed that only $0.023 \%$ of all consultations by men in the previous month were for pleuritic pain during the winter. Since $<2 \%$ of all those aged 40 years or more on the practice lists were asked to participate, it is most unlikely that their initial selection as potential controls was connected with whether they had had recent pleuritic pain. In addition, controls reported primary symptoms with the same frequency as cases. Overall, our results are consistent with those of the GPRD studies, in contrast with which our study had the advantage of direct clinical histories of respiratory infection from cases and controls. We conclude that recent respiratory infection manifested by chest pain or fever may be a factor leading to MI. Several different pathways may be involved, including increased thrombogenicity, atherogenesis, and plaque rupture. ${ }^{15}$ If recent respiratory infection contributes to MI, the implications include further encouragement of a

Abbreviations: $\mathrm{Cl}$, confidence interval; GPRD, General Practice Research Database; MI, myocardial infarction; OR, odds ratio 
Table 1 Odds ratios for myocardial infarction associated with symptoms of respiratory infection starting in the preceding month

\begin{tabular}{|c|c|c|c|c|c|c|c|}
\hline \multirow[b]{2}{*}{ Symptom } & \multirow[b]{2}{*}{ Cases $(n=119)$} & \multirow[b]{2}{*}{ Controls $(n=214)$} & \multicolumn{2}{|c|}{ Unadjusted analysis* } & \multicolumn{2}{|c|}{ Adjusted analysis $\dagger$} & \multirow[b]{2}{*}{$p$ Value } \\
\hline & & & OR & $95 \% \mathrm{Cl}$ & OR & $95 \% \mathrm{Cl}$ & \\
\hline \multicolumn{8}{|c|}{ Primary definition } \\
\hline No & $98(82 \%)$ & $182(85 \%)$ & 1 & NA & 1 & NA & \\
\hline Yes & $21(18 \%)$ & $32(15 \%)$ & 1.0 & 0.6 to 1.9 & 1.0 & 0.5 to 1.9 & 0.98 \\
\hline \multicolumn{8}{|c|}{ Chest pain on breathing } \\
\hline No & 98 (82\%) & 211 (99\%) & 1 & NA & 1 & NA & \\
\hline Yes & $21(18 \%)$ & $3(1 \%)$ & 17.8 & 4.1 to 77.2 & 17.5 & 3.6 to 85.6 & $<0.0001$ \\
\hline \multicolumn{8}{|l|}{ Fever } \\
\hline No & $96(81 \%)$ & 205 (96\%) & 1 & NA & 1 & NA & \\
\hline Yes & $23(19 \%)$ & $9(4 \%)$ & 4.1 & 1.9 to 9.0 & 5.9 & 2.0 to 16.8 & 0.0004 \\
\hline
\end{tabular}

*Except for the matching factors (age, sex, social class, calendar period).

†For the primary definition of infection: adjusted for history of angina and smoking status in addition to the matching factors; for chest pain on breathing and fever: adjusted for each other in addition to history of angina, smoking, and the matching factors.

$\mathrm{Cl}$, confidence interval; NA, not applicable; OR, odds ratio.

high and more consistent uptake of influenza immunisation by older people and by those in vulnerable groups, as well as aborting infections at an early stage.

\section{ACKNOWLEDGEMENTS}

We particularly thank Jo Molloy and Sarah Dawe for recruiting patients and interviewing them and the controls. We are grateful to the general practitioners for identifying controls. Staff in the former Medical Research Council Epidemiology and Medical Care Unit who established the age-sex registers for selecting controls and managed data processing were Ken Whyte and Wendy Quy. Claire Cooke worked on the required sample sizes. We are grateful to Dr Mary Thompson for help with the data from the IMS Disease AnalyserMediPlus database. Professor Rory Collins, Professor John Danesh, Dr Bianca de Stavola, Professor Desmond Julian, Professor Stuart Pocock, and Dr Liam Smeeth kindly commented on earlier drafts of the paper.

\section{Authors' affiliations}

T C Clayton, T W Meade, London School of Hygiene \& Tropical Medicine, London, UK

N E Capps, Princess Royal Hospital, Telford, UK

N G Stephens, Northwick Park Hospital, Harrow, UK

J A Wedzicha, St Bartholomew's and Royal London School of Medicine and Dentistry, London, UK

Role of the funding source: The Medical Research Council, who funded the study, had no role in the study design, in the collection, analysis, and interpretation of the data, in the writing of the report, or in the decision to submit the paper for publication.

Conflict of interest statement: The authors declare no conflict of interest with respect to this case-control study. However, Professor Tom Meade is a member of the IMS Health Independent Scientific Ethics Advisory Committee and receives payment for his work in this capacity.

Ethical approval: The study was approved by the Local Research Ethics Committees of the two hospitals (Northwick Park Hospital, Harrow, and the Princess Royal Hospital, Telford).

Correspondence to: Tim C Clayton, Medical Statistics Unit, London School of Hygiene \& Tropical Medicine, Keppel Street, London WC1E 7HT, UK; tim.clayłon@|shtm.ac.uk

Accepted 4 May 2005

\section{REFERENCES}

1 Danesh J, Collins R, Peto R. Chronic infections and coronary heart disease: is there a link? Lancet 1997;350:430-6.

2 Curwen M. Excess winter mortality: a British phenomenon? Health Trends 1990/91;22:169-75

3 Meier CR, Jick SS, Derby LE, et al. Acute respiratory-tract infections and risk of first-time acute myocardial infarction. Lancet 1998:351:1467-71.

4 Smeeth L, Thomas SL, Hall AJ, et al. Risk of myocardial infarction and stroke after acute infection or vaccination. N Engl J Med 2004;351:261 1-8.

5 Wedzicha JA, Seemungal TA, MacCallum PK, et al. Acute exacerbations of chronic obstructive pulmonary disease are accompanied by elevations of plasma fibrinogen and serum IL-6 levels. Thromb Haemost 2000;84:210-5. 